\title{
Targeting the gut to treat multiple sclerosis
}

\author{
Laura Ghezzi, ${ }^{1,2}$ Claudia Cantoni,, Gabriela V. Pinget, ${ }^{3}$ Yanjiao Zhou, ${ }^{4}$ and Laura Piccio ${ }^{1,5,6}$ \\ ${ }^{1}$ Department of Neurology, School of Medicine, Washington University in St. Louis, St. Louis, Missouri, USA. ${ }^{2}$ University of Milan, Milan, Italy. ${ }^{3}$ Charles Perkins Centre, Faculty of Medicine and Health, \\ University of Sydney, Sydney, New South Wales, Australia. ${ }^{4}$ Department of Medicine, School of Medicine, UConn Health, Farmington, Connecticut, USA. ${ }^{5}$ Brain and Mind Centre, School of Medical Sciences, \\ University of Sydney, Sydney, New South Wales, Australia. ${ }^{6}$ Hope Center for Neurological Disorders, Department of Neurology, School of Medicine, Washington University in St. Louis, St. Louis, Missouri, USA.
}

\begin{abstract}
The gut-brain axis (GBA) refers to the complex interactions between the gut microbiota and the nervous, immune, and endocrine systems, together linking brain and gut functions. Perturbations of the GBA have been reported in people with multiple sclerosis (pwMS), suggesting a possible role in disease pathogenesis and making it a potential therapeutic target. While research in the area is still in its infancy, a number of studies revealed that pwMS are more likely to exhibit altered microbiota, altered levels of short chain fatty acids and secondary bile products, and increased intestinal permeability. However, specific microbes and metabolites identified across studies and cohorts vary greatly. Small clinical and preclinical trials in pwMS and mouse models, in which microbial composition was manipulated through the use of antibiotics, fecal microbiota transplantation, and probiotic supplements, have provided promising outcomes in preventing CNS inflammation. However, results are not always consistent, and large-scale randomized controlled trials are lacking. Herein, we give an overview of how the GBA could contribute to MS pathogenesis, examine the different approaches tested to modulate the CBA, and discuss how they may impact neuroinflammation and demyelination in the CNS.
\end{abstract}

\section{Introduction}

The gut-brain axis (GBA) is the bidirectional communication between the CNS and the gastrointestinal system, linking brain and gut functions. It involves a complex network of interactions between the endocrine, immune, autonomic, and enteric nervous systems. The gut microbiota and the intestinal barrier, key players in the GBA, have recently attracted much interest due to their emerging role in mediating health and disease and potential use as therapeutic targets. The gut microbiota affects many aspects of brain development and function, including microglia and astrocyte maturation and polarization, blood-brain barrier (BBB) formation and permeability, neurogenesis, and myelination (1-7). GBA disruption may participate in the pathophysiology of several brain disorders, including multiple sclerosis (MS) (6, 8-10). Numerous reports in the last decade have focused on the role of the gut microbiota and intestinal barrier in MS and its main animal model, experimental autoimmune encephalomyelitis (EAE). However, a great deal of controversy exists surrounding the extent and the exact mechanisms through which altered GBA may influence the development of CNS inflammation, demyelination, and axonal loss. In this Review, we summarize current literature exploring the GBA's role in MS and relevant animal models, focusing on current and potential therapeutic strategies targeting the GBA to improve disease in people with MS (pwMS).

\section{The GBA in MS and its animal models}

MS is a multifactorial disease arising from a complex interplay between genetic and environmental factors $(11,12)$. Alterations in

Conflict of interest: LP receives research support from Alector. Copyright: (5) 2021, American Society for Clinical Investigation. Reference information: J Clin Invest. 2021;131(13):e143774. https://doi.org/10.1172/JCl143774. gut microbiota composition (13-18), gut-derived products (19-21), intestinal permeability (22-24), and endocrine (25) and enteric nervous system functions (26) have been described in pwMS. The consequences of these disruptions are linked directly or indirectly to activation of the immune system against CNS self-components and development of CNS autoimmunity. These pathophysiological aspects have started to be characterized in MS animal models, whereas mechanistic studies in pwMS are still scarce. Below, we will consider the role of the different GBA components (gut microbiota, intestinal barrier, and immune, autonomic, and endocrine systems) in CNS autoimmunity and evidence of their alterations in pwMS and in MS animal models.

\section{The gut microbiota and products of bacterial metabolism}

Previous studies have described perturbations in both gut microbiota composition and gut-related metabolic pathways in pwMS $(13-21,27)$. Differences in single taxonomic units are more widely reported than large-scale gut microbiota differences when comparing pwMS with healthy controls (HCs) $(13,14,16,28)$. Unfortunately, results across studies lack consistency. Sample size, subject heterogeneity, study design, type of controls, geographical location, sequencing platforms, and regions of 16S rRNA gene sequencing may all contribute to lack of reproducibility (29). Despite this limitation, certain taxa are consistently noted as differentially represented in pwMS compared with HCs. In a study involving 34 monozygotic twins discordant for MS, the genus Akkermansia was overrepresented in the untreated MS twin compared with the healthy twin (16). Two other studies confirmed these results, also demonstrating an increase in Acinetobacter and Methanobrevibacter and a decrease in Parabacteriodes and Butyricimonas genera $(13,17)$. Other consistent changes observed across 


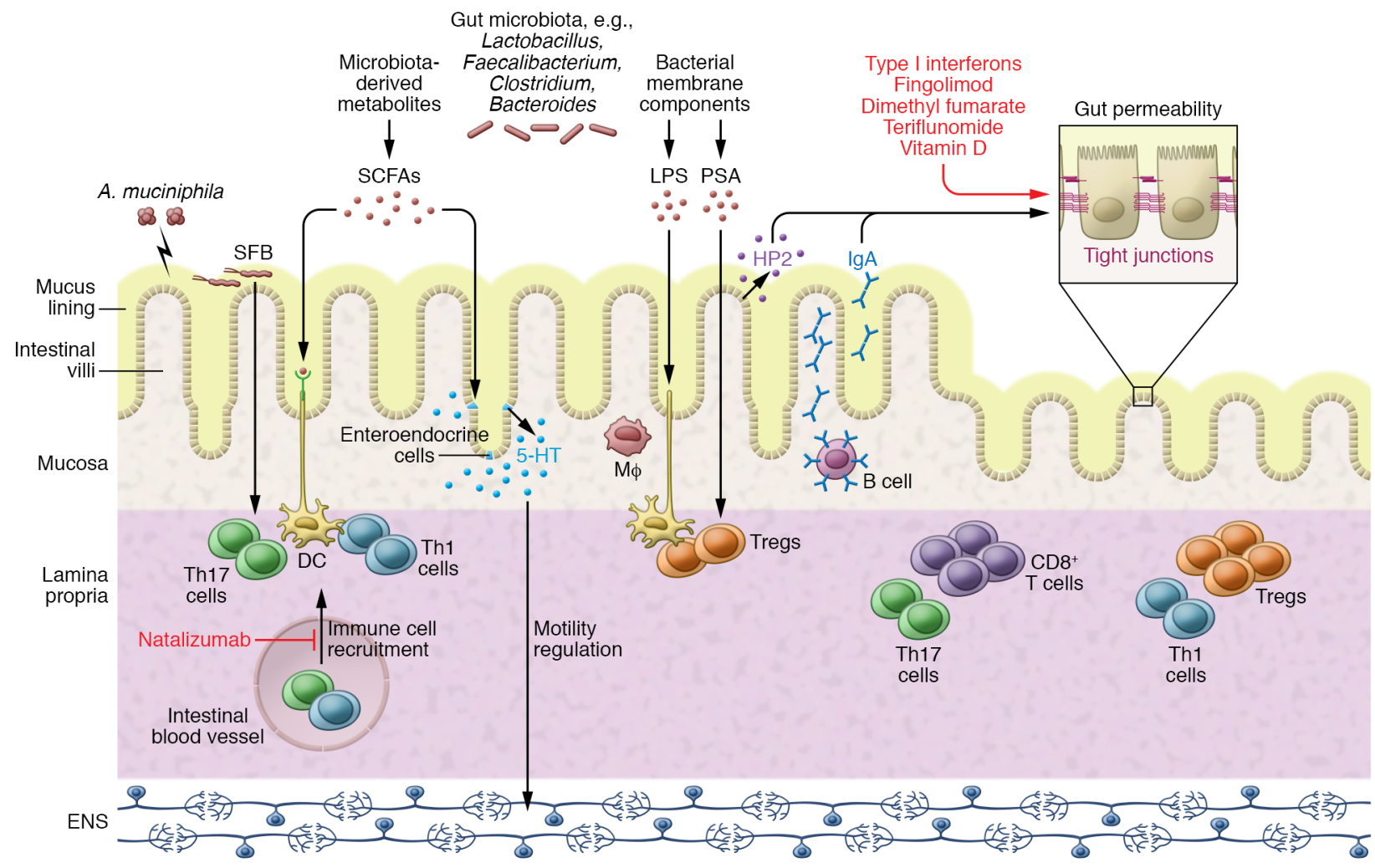

Figure 1. Gut-immune system interactions. The gut microbiota interacts with both the enteric nervous system and the immune system through multiple mechanisms. Bacterial-derived metabolites (i.e., SCFAs and bile acids) or membrane components (i.e., LPS and polysaccharide A [PSA], the latter associated with the membrane of Bacteroides fragilis) can modulate the maturation and development of Th17 cells and Tregs and can regulate intestinal motility. Tight junctions on epithelial cells play a critical role in controlling intestinal permeability, and their function can be regulated by epithelial molecules (i.e., IgA and zonulin [HP2]) that can also impact gut microbiome composition. Disease-modifying treatments and vitamin D are known to act on gut permeability, including T cell migration through intestinal vessels (i.e., effects of natalizumab, a monoclonal antibody widely used to treat MS). The effector mechanisms depicted in the figure could act differently in the small intestine versus the colon. For instance, SCFAs are preferentially produced by fermentation of dietary fibers in the colon, where they can influence Treg differentiation. In contrast, segmented filamentous bacteria (SFB) are commensal bacteria attached to the ileal epithelium that in mice have been shown to promote the differentiation of Th17 cells. 5-HT, 5-hydroxytryptamine; A. muciniphila, Akkermansia muciniphila; DMF, dimethyl fumarate; ENS, enteric nervous system.

multiple studies included a reduced abundance of Bacteroidaceae family, Faecalibacterium, Clostridium species, and Prevotella strains $(15,27,30,31)$. Clostridium species were shown to promote Treg accumulation in the colon, with a consequent immunomodulatory effect (32). Polysaccharide A, from the capsule of the human commensal Bacteroides fragilis, has been described as a powerful immune cell activator that induced clonal $\mathrm{CD} 4^{+} \mathrm{T}$ cell expansion (33) and IL-10 secretion in T and B cells $(34,35)$. Whether these gut microbiota alterations in MS could contribute to disease pathogenesis or are just a consequence remains unknown. Supporting a real pathogenic role is the finding that gut microbiota or gut-derived molecules obtained from pwMS could modulate EAE when transferred into mice $(16,17,36)$.

Microbial metabolites are altered in pwMS. Pathways most commonly found modulated in the gut microbiota of pwMS include carbohydrate and lipid metabolism and short chain fatty acid (SCFA) and bile acid synthesis $(14,37)$. SCFAs, including acetate, propionate, and butyrate, are produced by microbial fermentation of dietary fiber in the colon, where they maintain intestinal barrier integrity and dampen intestinal inflammation (38). They are then absorbed by the host and exert systemic immunomodula- tory effects $(20,38,39)$. Emerging evidence indicates that SCFAs can cross the BBB and control neuroimmune homeostasis (40). SCFAs were significantly decreased in people with secondary progressive MS with long disease duration compared with HCs (20). Propionate levels were reduced in serum and stool samples of two different cohorts of pwMS across different disease subtypes compared with HCs. This alteration was concomitant to a reduction in the abundance of SCFA-producing gut bacteria, decreased Tregs, and increased IL-17-producing T cell proportions in blood (21). In contrast, plasma levels of acetate were reported to be higher in a cohort of pwMS compared with HCs and correlated with both Expanded Disability Status Scale (EDSS) score and proportions of IL-17-producing CD8 ${ }^{+}$cells (41).

Primary bile acids are mainly produced by the liver and subsequently modified by intestinal microbes to produce secondary bile acids. Bile acids and their metabolites can modulate glial and myeloid cell activation within the CNS via cell receptors and may play a neuroprotective role (42). Circulating levels of bile acid metabolites are altered in pediatric and adult cohorts of pwMS, and in EAE mice (19,43); indeed, Bhargava and colleagues identified dysregulation of secondary bile acid metabolism in pwMS (19). 


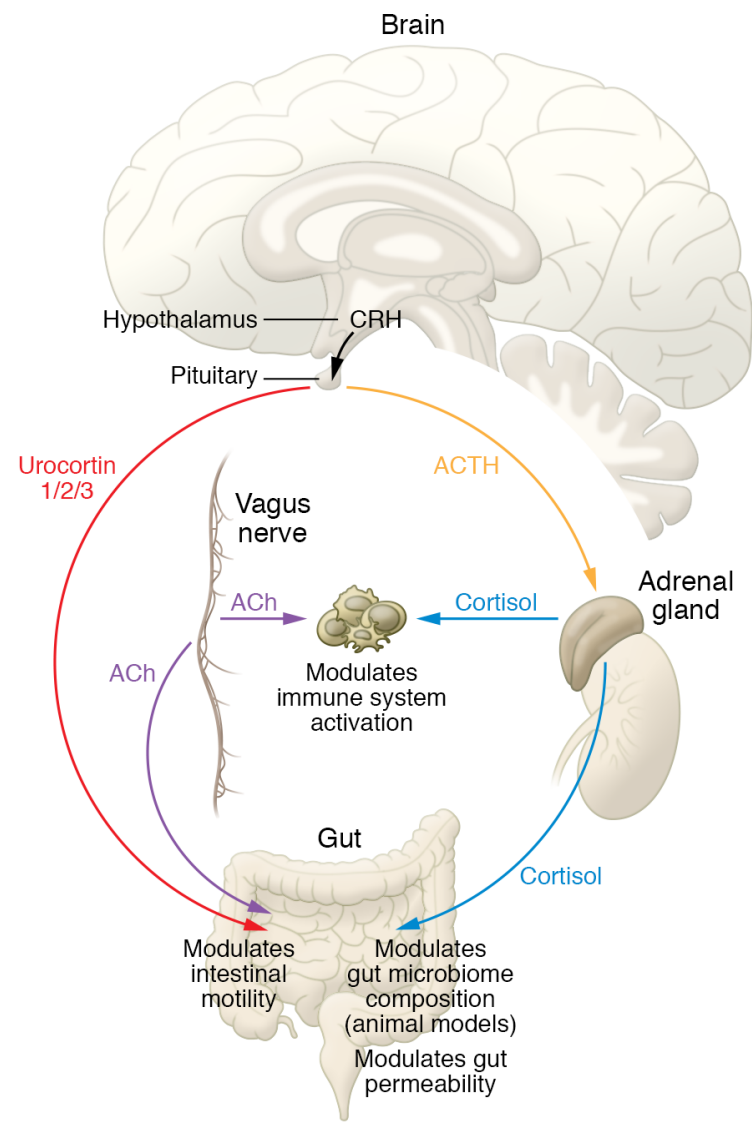

Figure 2. Gut-brain communications through the endocrine system and vagus nerve. The hypothalamic-pituitary-adrenal (HPA) axis modulates intestinal motility (directly through urocortin production), gut microbiome composition, intestinal permeability, and immune system activation (through cortisol production). The vagus nerve influences the immune system through acetylcholine production and intestinal permeability and motility directly and through the ENS. ACTH, adrenocorticotropic hormone; $\mathrm{CRH}$, corticotropin-releasing hormone.

Mice supplemented with the secondary bile acid tauroursodeoxycholic acid (TUDCA) had lower EAE disease burden compared with untreated mice, indicating this bacterial product's protective role.

\section{The intestinal barrier}

The intestinal barrier's critical role in absorbing nutrients and metabolites, while preventing the permeation of intestinal microbes, toxins, and other antigens (44), is achieved through the actions of key components: the intestinal immune system; the epithelial layer, which regulates paracellular permeability or "gut leakiness"; and secretory products, including the mucus lining, which creates physical separation between the epithelium and luminal contents, and IgA, which is carried along the mucus lining $(24,45)$ (Figure 1).

The intestinal epithelium consists of a single layer of highly specialized cells held together by more than 50 transmembrane tight junction (TJ) proteins $(45,46)$. TJ proteins form a physical barrier between the apical and basolateral epithelial compartments and selectively regulate passive diffusion of ions and water-soluble molecules through the paracellular pathway (47).
The three major TJ proteins are occludin, claudins, and junctional adhesion molecule proteins (48). TJ barriers are dynamic, and $\mathrm{TJ}$ protein expression is influenced by many factors, including age, microbial antigens, and certain cytokines such as TNF- $\alpha$ (49-52). In human small intestine, the secreted protein zonulin or haptoglobin 2 precursor (HP2) is a master regulator of TJ protein expression, and its presence triggers TJ disassembly (47). Heightened intestinal permeability or "leaky gut" can cause unregulated passage of luminal contents into the host (47) and subsequent activation of immune cells by otherwise nonimmunogenic commensal microbes. Indeed, a leaky gut is a common hallmark of inflammatory disease, and HP2 overexpression has been observed in multiple autoimmune diseases, including MS $(23,47,53)$.

A recent pilot study demonstrated that people with relapsing-remitting MS (RRMS) are more likely to have compromised intestinal permeability (54). Plasma from pwMS displays higher biomarkers of intestinal barrier integrity, particularly HP2 (55). These changes correlate with BBB disruption, as measured by MRI (55). Interestingly, HP2 is thought to pass into the bloodstream to reach the $\mathrm{BBB}$, providing a possible mechanistic explanation for how intestinal barrier integrity may influence BBB permeability and subsequently CNS inflammation $(24,55)$. However, increased BBB permeability by HP2 was demonstrated only in vitro (24).

Consistent with altered gut barrier integrity, pwMS have increased low-grade bacterial translocation as measured by detectable plasma levels of LPS $(23,47)$ and increased LPS-binding protein, which was also reported in EAE $(55,56)$. More recently, peptidoglycan, an abundant bacterial cell wall component, was detected in brain tissue lesions of pwMS $(57,58)$. However, whether its presence in blood is indicative of inflammatory disease is not understood since peptidoglycan was previously detected in the serum of healthy patients (57).

As in pwMS, EAE mice develop increased intestinal barrier permeability accompanied by morphological changes in the small intestine $(22,59)$. These intestinal alterations were observed after adoptive transfer of myelin oligodendrocyte glycoprotein-reactive (MOG-reactive) $\mathrm{CD} 4^{+} \mathrm{T}$ cells, suggesting that circulating autoreactive $\mathrm{T}$ cells are critical to induce gut barrier alterations (22). Further, loss of intestinal barrier integrity worsened at EAE day 15 versus day 7, suggesting that EAE progression promotes gut permeability (22).

The intestinal tract's mucus lining is a critical physical barrier between the host and microbes (60), composed of glycosylated proteins, collectively termed mucins, and electrolytes, lipids, and IgA (60). Mucin loss or excessive mucin degradation has been demonstrated to increase gut permeability and thereby create an inflammatory state. Certain microbes, such as A. muciniphila, are known to degrade the mucus layer and thereby alter the local immune milieu (61). As outlined above, A. muciniphila is increased in both pwMS and EAE mice $(13,62)$. While a strong correlation exists between the presence of mucus-degrading bacteria and MS, its contribution to disease pathogenesis remains unknown. Mucus-degrading bacteria may facilitate the inflammatory effects of other microbes and therefore only wreak havoc when combined with certain immunogenic bacteria (63). In contrast, a study in which miR-30d transfer promoted Akkermansia abundance found that EAE symptoms were suppressed via Treg promotion (36). Consistent with this, a recent study associated Akkermansia with lower disability in pwMS, and 


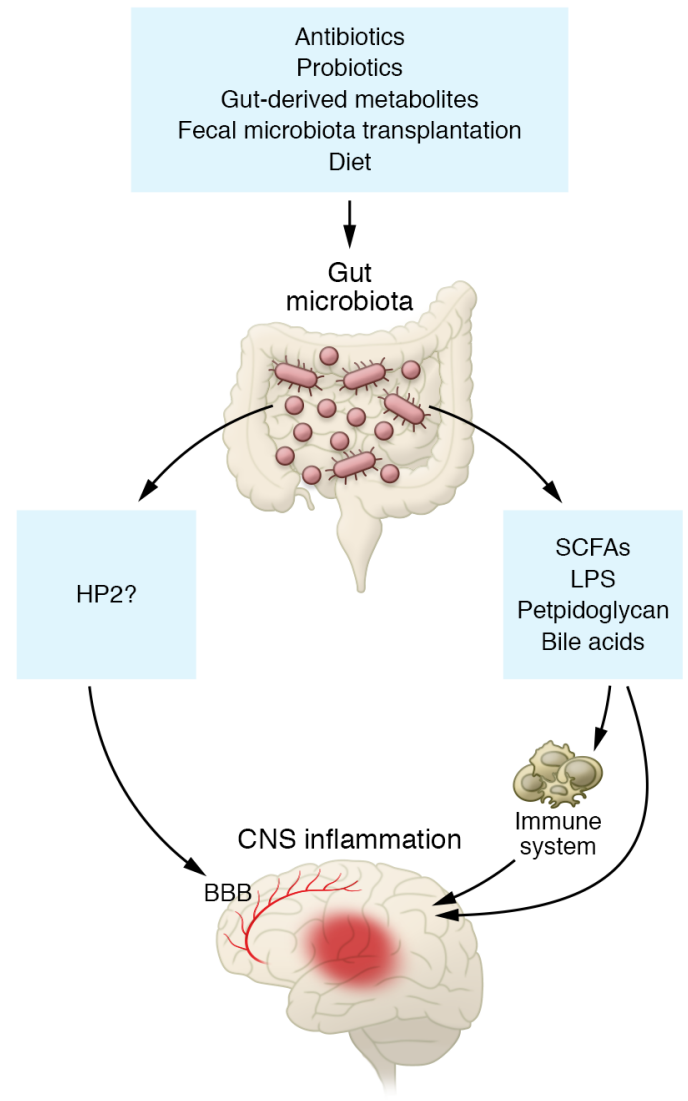

Figure 3. Gut-based therapies to modulate CNS autoimmunity through the GBA. Antibiotics, probiotics, gut-derived metabolites, fecal microbiota transplantation (FMT), and diet are all possible therapeutic approaches that could modulate CNS inflammation through the GBA. As suggested by preclinical and clinical studies, gut-based therapies can modify gut microbiota composition, which in turn modulates the production of gut-derived products and metabolites (e.g., LPS, SCFAs, peptidoglycan, and bile acids), acting on both the immune system and the CNS. Zonulin (HP2), produced in the small intestine, where it modulates gut barrier integrity, was suggested to have a direct effect also on BBB permeability.

when transferred in mice, Akkermansia ameliorated EAE via reduction of ROR $\gamma \mathrm{t}^{+}$and IL-17-producing $\gamma \delta \mathrm{T}$ cells (64), suggesting an overall beneficial role similar to that observed in obesity, diabetes, and aging (65). Therefore, it is possible that Akkermansia's effects on health and diseases are context dependent and should be considered within the specific disease setting and parallel changes of other bacteria and gut microbiome structure.

\section{The intestinal immune system}

The mammalian gastrointestinal tract (GIT) harbors the body's greatest proportion of immune cells (66). These cells are in close contact with local microbiota and have the difficult task of protecting against pathogens while remaining tolerant toward food antigen and nonpathogenic commensals. The intestinal immune system comprises innate and adaptive immune cells, detailed extensively elsewhere $(66,67)$ (Figure 1). The microbial environment critically shapes the local immune system, as revealed by the vastly diminished immune compartment observed in the GIT of germ-free (GF) mice (67-69). In normal conditions, the intestinal lamina propria (LP) harbors a high proportion of Th17 cells (70), which protect against pathogens, while
Tregs attenuate inflammatory responses and initiate immune tolerance $(71,72)$. It is thought that an imbalance between Tregs and Th17 cells may contribute to the breakdown of immune tolerance, driving a number of autoimmune diseases, including MS and EAE (73-75).

The microbiota can influence activation and proliferation of Tregs and Th17 cells, and both GF and antibiotic-treated mice develop attenuated $\operatorname{EAE}(76,77)$. In one study, recolonization of GF mice with commensal bacteria restored EAE susceptibility by promoting differentiation of Th17 cells in the gut, and recolonization with segmented filamentous bacteria alone was enough to promote EAE development via stimulation of IL-17 production (75). People with active RRMS had significantly higher percentages of Th17 cells in the small intestinal mucosa compared with HCs (31). In EAE, MOG-specific Th17 cells migrate to the colonic LP before disease onset and induce dysbiosis; in this study, blocking intestinal homing of Th17 cells attenuated disease severity (78). New research has revealed that IL-17/- mice have a distinct microbiota, which protects from EAE development. However, this protection was lost following transfer of microbiota from WT mice, indicating that intestinal IL-17 production might directly influence gut microbiome composition and be necessary for disease development (75). In contrast, another study found that blocking MOG-specific Th17 cells' migration to the gut worsened disease severity in a mouse model of spontaneous EAE. This study's authors concluded that these cells were no longer sequestered in the intestine and so continued to contribute to disease progression within the CNS (79).

Humoral immunity also plays a paramount role in the gut. Gut microbiota influence differentiation of $\operatorname{IgA}^{+}$plasma cells (PCs), and IgA binding of specific bacteria was shown to influence gut colonization and niche formation in the human colon (80). Accordingly, GF mice have very low IgA levels (81). In a recent paper, pwMS exhibited a reduction in fecal IgA during MS relapses, and $\operatorname{IgA}^{+} B$ cells were able to gain access to the inflamed CNS (82). $\mathrm{IgA}^{+} \mathrm{B}$ cell removal exacerbated EAE, an outcome rescued by $\mathrm{PC}$ transfer, demonstrating their protective role (82). Moreover, gut microbiota-specific IgA ${ }^{+} \mathrm{B}$ cells showed a different specificity in pwMS compared with HCs in another recent paper: the most prominent operational taxonomic units bound by IgA in pwMS, but not in HCs, were A. muciniphila, Eggerthella lenta, Bifidobacterium adolescentis, and Ruminococcus (83).

\section{The autonomic and enteric nervous systems}

The autonomic nervous system comprises the sympathetic, parasympathetic, and enteric divisions, which together connect the gut and the CNS $(84,85)$. The ENS consists of neurons and glial cells located in the gut smooth muscle and submucosal layers. These cells can regulate intestinal motility and permeability independently of CNS inputs. Gut microbiota influence both CNS and ENS development, as evident in GF mice, whose myenteric plexus is structurally abnormal, exhibiting decreased nerve density and number of neurons per ganglion (86). Some neurotransmitters are common between the CNS and ENS, and perturbations of the ENS have been described in neuroinflammatory and neurodegenerative diseases $(8,26,85)$. In a B cell/antibody-dependent EAE model, ENS degeneration in the myoenteric plexus was described before disease onset and was mediated by autoantibodies (26), which were also detected in a small cohort of pwMS, along with ENS gliosis (26). 
Table 1. Preclinical studies testing antibiotic therapy to modulate CNS autoimmunity

\begin{tabular}{|c|c|c|c|c|}
\hline Animal model & Intervention & Results & Proposed mechanisms & Ref. \\
\hline $\begin{array}{l}\text { EAE } \\
\text { SJL mice }\end{array}$ & $\begin{array}{l}\text { Oral ampicillin, vancomycin, neomycin, and } \\
\text { metronidazole for } 7 \text { days before EAE induction }\end{array}$ & Disease amelioration & Accumulation of Tregs in peripheral lymph nodes & 108 \\
\hline $\begin{array}{l}\text { EAE } \\
\text { C56BL/6 mice }\end{array}$ & $\begin{array}{l}\text { Intraperitoneal ampicillin treatment in } \\
\text { late-stage EAE }\end{array}$ & Disease worsening & $\begin{array}{l}\downarrow \text { commensal bacteria capable of transforming } \\
\text { tryptophan into AHR agonists }\end{array}$ & 113 \\
\hline $\begin{array}{l}\text { EAE } \\
\text { NOD/Shilt mice }\end{array}$ & Oral antibiotic mix before EAE induction & Disease amelioration & $\begin{array}{l}\text { Altered gut microbiome and increased Tregs } \\
\text { in Peyer patches }\end{array}$ & 110 \\
\hline $\begin{array}{l}\text { EAE } \\
\text { C56BL/6 mice }\end{array}$ & $\begin{array}{l}\text { Oral norfloxacin and Clostridium butyricum } \\
\text { (C. butyricum) for } 7 \text { days before EAE induction }\end{array}$ & Disease amelioration & $\begin{array}{l}\downarrow \text { Firmicutes/Bacteroidetes ratio and Th17, while } \\
\text { increasing Treg responses in the GIT }\end{array}$ & 30 \\
\hline $\begin{array}{l}\text { Lysolecithin-induced } \\
\text { CNS demyelination } \\
\text { C57BL/6 mice }\end{array}$ & $\begin{array}{l}\text { Oral treatment with antibiotic mix for } 12 \text { weeks } \\
\text { before lysolecithin }\end{array}$ & $\begin{array}{l}\uparrow \text { inflammatory activation of infiltrating } \\
\text { macrophages and resident microglia; impaired } \\
\text { myelin debris clearance and OPC differentiation }\end{array}$ & Altered gut microbiota composition & 114 \\
\hline
\end{tabular}

AHR, aryl hydrocarbon receptor; OPC, oligodendrocyte precursor cell.

The vagus nerve is the principal component of the parasympathetic nervous system. Its afferent and efferent fibers innervate the GIT and communicate directly and indirectly with the gut microbiota and the immune system. Vagal afferent fibers can sense bacterial products such as LPS and hormones (e.g., cholecystokinin, glucagon-like peptide-1, peptide YY) produced by enteroendocrine cells in response to food intake or microbial metabolites $(87,88)$. Acetylcholine (ACh), the neurotransmitter released by vagus efferent fibers, was shown in vitro to inhibit proinflammatory cytokine production in macrophages, leading to the "cholinergic antiinflammatory hypothesis" (89). Interestingly, people with RRMS have reduced ACh both in serum and cerebrospinal fluid (CSF), along with increased inflammatory cytokines IL-1 $\beta$ and IL-17 $(90,91)$. Consistent with this, increased ACh levels induced by treatment with acetylcholinesterase inhibitors in mice with EAE resulted in suppression of $\mathrm{T}$ cell activation and decreased disease severity (92). Similarly, another study found that vagotomy also ameliorated EAE by inhibiting proliferation and differentiation of $\mathrm{CD} 4^{+} \mathrm{T}$ cells by reducing $\mathrm{ACh}$ and lymphokine secretion in the cortex (93). ACh's seemingly contradictory role in regulating inflammation could reflect opposing effects of ACh receptors (muscarinic vs. nicotinic) expressed on immune cells and highlights the need for further research in the area.

\section{The endocrine system}

The endocrine system is another pathway of communication between the gut and the CNS (Figure 2). The HPA axis is considered a major neuroendocrine organ, regulating a multitude of body functions. In situations of stress, CRH, produced by the hypothalamus, induces the release of ACTH by the pituitary gland, which determines the release of corticosteroids by the adrenal gland (94). CRH and related hormones urocortin 1-3 influence intestinal motility, permeability, and immune responses (95-97). Evidence of a link between the GBA and HPA axes is derived, again, from experiments using GF mice. GF mice exhibited exaggerated HPA axis activation in response to stress that was not reverted upon recolonization in adult mice (3). Emerging evidence indicates the existence of bidirectional communication between the neuroendocrine system and gut microbiota (98). The presence of gut microbiota influenced not only the physiology of the HPA axis but also its response to stress, possibly through the modulation of vagal responses $(99,100)$. Altered levels of brain-derived neurotrophic factor (BDNF), NMDA, and 5-HT expression in the cerebral cortex, amygdala, and hippocampus have been described in GF mice (101). Several lines of evidence implicate the HPA axis in MS pathogenesis, MS disease progression, and occurrence of comorbid mood disorders (102-104). Clinical and postmortem studies in pwMS demonstrated elevated basal cortisol plasma levels, enlarged adrenal glands, higher cortisol levels in CSF, and increased CRH-producing hypothalamic neurons (104-106). Notably, low HPA axis activity was correlated with increased disease severity in pwMS (25).

\section{Targeting the gut to modulate CNS autoimmunity}

Animal studies clearly support the involvement of gut microbiota in regulating CNS inflammation, microglia functions, myelination, and BBB integrity $(1,2,107)$. Since these processes have proven relevant in the pathophysiology of CNS autoimmunity, perturbations of the gut microbiota and/or gut permeability are expected to impact the pathology and clinical course of disease in mice and possibly in humans. Accordingly, various interventions targeting gut microbiota composition and/or intestinal integrity have been implemented to treat CNS autoimmunity in preclinical and clinical studies (Figure 3). Most studies evaluating the effects of gut manipulation on CNS inflammation have been performed in the EAE model; however, a handful of studies have used other experimental MS models, such as the Theiler's murine encephalomyelitis virus 
Table 2. Preclinical studies testing probiotic supplementation to modulate CNS autoimmunity

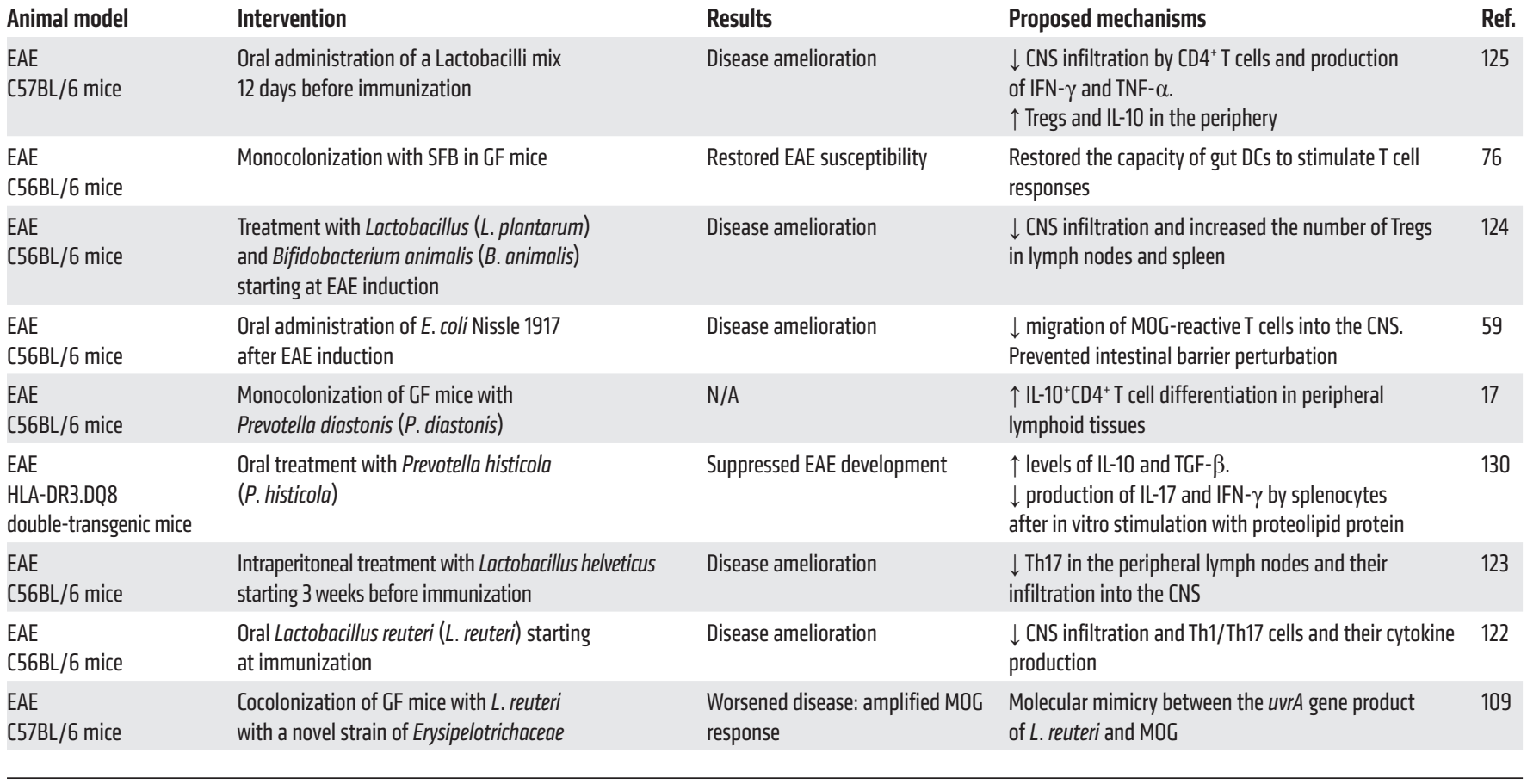

(TMEV) model and toxin-induced models of CNS demyelination. Tables 1, 2, 3, and 4 summarize preclinical and clinical studies targeting the gut to modulate CNS autoimmunity.

Antibiotic treatment. Antibiotic treatment has been shown to ameliorate EAE through effects mediated via the gut microbiota $(30,77,108-110)$. Seminal work demonstrated that oral treatment with broad-spectrum antibiotics significantly altered the gut flora and reduced EAE severity in a Treg-dependent manner (108). Disease amelioration did not occur when antibiotics were given intraperitoneally, thereby bypassing the gut, suggesting that modulation of the gut microbiota produces protective effects. Similarly, Yokote and colleagues reported that antibiotic treatment protected mice from EAE but found invariant NK T cells as the key drivers (77). A recent report found that oral treatment with ampicillin decreased EAE severity and identified two molecules produced by $L$. reuteri and a newly isolated strain from the Erysipelotrichaceae family, which act synergistically to induce accumulation of MOG-specific Th17 cells in the small intestine (109). Further, several studies have reported that treatment with antibiotics before EAE induction or at presymptomatic stages, but not after disease onset, protected mice from disease $(30,110,111)$. Antibiotics administered at a presymptomatic phase also protected from motor dysfunction in TMEV-infected mice, another animal model of MS (112). Indeed, one study reported that ampicillin treatment during clinical EAE worsened the disease, whereas vancomycin treatment exerted no clinical effect (113). Effects of antibiotic treatment were also studied in the lysolecithin-induced model of CNS demyelination. In this study, antibiotic treatment before injection of the toxic compound into the spinal cord resulted in increased immune response 14 days after demyelination induction, with impaired debris clearance, without affecting remyelination (114).
Studies focusing on the effects of antibiotic treatment in pwMS are scarce. Two small studies investigating the effects of doxycycline combined with IFN- $\beta$ in pwMS reported decreased relapse rates, improved measures of disability, and reduced gadolinium-enhancing lesions compared with IFN- $\beta$ treatment alone $(115,116)$. Another randomized, double-blind placebo-controlled study showed that minocycline delayed the conversion of people with CIS to MS over a 6-month but not 24-month period (117). A larger study on people with CIS is ongoing (ClinicalTrials.gov NCT04291456).

In summary, EAE studies suggest that antibiotic treatment has beneficial effects when administered preventatively but not once disease is established. Mechanisms mediating antibiotics' effects are not understood but likely are due to microbial modulation, which may in turn alter immune activation $(111,118)$. Long-term antibiotic therapy in pwMS shows promising results but carries risks, such as promoting the growth of opportunistic pathogens, including Clostridium difficile (C. difficile), fungi, and antibiotic-resistant infections (119). Furthermore, the possibility of direct side effects, such as dental discoloration, rash, and photosensitivity with minocycline treatment (117), must also be considered.

Probiotic supplementation. Probiotics have gained much interest recently as potential therapeutic agents in MS. These live microorganisms are believed to exert their effects by modulating the gut flora to one that promotes intestinal barrier integrity and differentiation and activation of immunoregulatory over inflammatory cell subsets $(120,121)$. Probiotic administration in rodents with EAE has focused mainly on the effects of Lactobacillus strains, either alone or in combination with other strains (122128). Most studies report an attenuation of EAE clinical course, but results are inconsistent and often contradictory. Herein we 
Table 3. Preclinical studies testing SCFA supplementation, FMT, and diet modifications to modulate CNS autoimmunity

\begin{tabular}{|c|c|c|c|c|c|}
\hline Intervention & Animal model & Intervention & Results & Proposed mechanisms & Ref. \\
\hline \multirow[t]{3}{*}{ SCFA } & $\begin{array}{l}\text { EAE } \\
\text { [57BL/6 mice }\end{array}$ & $\begin{array}{l}\text { Supplementation with LCFAs or SCFAs } \\
\text { at immunization or at disease onset }\end{array}$ & $\begin{array}{l}\text { Treatment with LCFAs exacerbated and } \\
\text { treatment with SCFAs ameliorated EAE }\end{array}$ & $\begin{array}{l}\text { LCFAs induced mainly Th1/Th17 cells } \\
\text { SCFAs induced Tregs }\end{array}$ & 136 \\
\hline & $\begin{array}{l}\text { EAE } \\
\text { [57BL/6 mice }\end{array}$ & $\begin{array}{l}\text { Oral administration of SCFAs for } 3 \text { weeks } \\
\text { before immunization }\end{array}$ & Disease amelioration & $\begin{array}{l}\uparrow \text { proportion of Tregs in the spleen } \\
\text { and peripheral lymph nodes }\end{array}$ & 137 \\
\hline & $\begin{array}{l}\text { EAE } \\
\text { C57BL/6 mice }\end{array}$ & $\begin{array}{l}\text { Oral administration of SCFAs for } 2-4 \text { weeks } \\
\text { before immunization }\end{array}$ & Disease amelioration & $\begin{array}{l}\downarrow \text { expression of proinflammatory } \\
\text { cytokines } \\
\uparrow \text { expression of IL-10 in the spinal cord }\end{array}$ & 20 \\
\hline \multirow{2}{*}{ FMT } & $\begin{array}{l}\text { EAE } \\
\text { [57BL/6 mice }\end{array}$ & $\begin{array}{l}\text { Colonization with microbiota from } \\
\text { pwMS } 6 \text { weeks before immunization }\end{array}$ & Disease worsening & $\begin{array}{l}\text { Lack of IL-10+ Treg induction in } \\
\text { mesenteric lymph nodes }\end{array}$ & 17 \\
\hline & $\begin{array}{l}\text { EAE } \\
\text { C56BL/6 mice }\end{array}$ & $\begin{array}{l}\text { Oral treatment with miR-30d after } \\
\text { EAE onset }\end{array}$ & Disease amelioration & $\begin{array}{l}\uparrow \mathrm{CD}^{+}{ }^{+} \mathrm{FoxP}^{+} \mathrm{T} \text { cells and MOG-specific } \\
\text { Tregs in the spleen }\end{array}$ & 36 \\
\hline \multirow{2}{*}{ Diet modification } & $\begin{array}{l}\text { EAE } \\
\text { C56BL/6 mice }\end{array}$ & $\begin{array}{l}\text { Tryptophan-free diet started } \\
\text { at the day of immunization }\end{array}$ & Prevented EAE & $\begin{array}{l}\downarrow \text { MOG-reactive CD4 }{ }^{+} \mathrm{T} \text { cells } \\
\text { and IL-17A; altered microbiome } \\
\text { composition }\end{array}$ & 153 \\
\hline & $\begin{array}{l}\text { EAE } \\
\text { [57BL/6 mice }\end{array}$ & $\begin{array}{l}\text { Methionine restriction started } 2 \text { weeks } \\
\text { prior to immunization }\end{array}$ & Disease amelioration & $\downarrow$ the expansion of Th17 cells & 154 \\
\hline
\end{tabular}

IF, intermittent fasting; LCFA, long-chain fatty acid; RR, relapsing-remitting.

discuss some of the main published studies, mindful that this is not a comprehensive review of the literature on this topic (121). Daily oral administration of a mixture of Lactobacillus strains in EAE was effective both at preventing disease development and reversing established disease, an outcome that was IL-10 dependent and correlated with Treg induction in mesenteric lymph nodes and the CNS (125). Similarly, treatment with a mixture of L. plantarum of human origin and B. animalis attenuated EAE clinically and induced Tregs in lymph nodes and spleen (124). Oral administration of human-derived L. reuteri after immunization ameliorated EAE with a decrease in Th1/Th17 subsets and related cytokines (122). In contrast, a recent study showed that L. reuteri administration amplified MOG-specific responses in GF mice monocolonized with a novel strain of the Erysipelotrichaceae family (109). The molecular similarity observed between MOG and the uvrA gene product of $L$. reuteri was advocated as the possible mechanism mediating EAE exacerbation (109). Similarly, another study found that $L$. reuteri exacerbated EAE in genetically susceptible mice (129). Such conflicting findings may result from $L$. reuteri's interaction with other commensal microbes present and their combined impact. Besides Lactobacillus strains, other gut bacteria given orally have been associated with probiotic effects and EAE amelioration, such as C. butyricum (30), E. coli strain Nissle 1917 (59), and P. histicola (130). Administration of a mixture of probiotics (Streptococcus, Bifidobacterium, and Lactobacillus strains) was also tested in the TMEV infection model, with beneficial effects of decreased CNS inflammation and increased motor activity during the disease (131).
Human studies are scarce, and treatment with a probiotic mix has only been tested in three studies involving pwMS (132-134). In two small double-blinded randomized controlled trials (RCTs), pwMS who received a mix of Lactobacillus and Bifidobacterium daily for 12 weeks showed significant improvements in disability score, depression, anxiety, and inflammatory markers, including reduced IL- 8 and TNF- $\alpha$ expression in PBMCs $(132,133)$. Similarly, Tankou and collaborators administered a probiotic mix containing Lactobacillus, Bifidobacterium, and Streptococcus to pwMS and HCs twice daily for 2 months and found reduced CD80 expression on peripheral monocytes. The described changes in gut microbiota composition and the immune system were not maintained after probiotic discontinuation, thus suggesting the necessity of continuous supplementation (134).

In summary, research into probiotic supplementation in EAE and MS remains inconclusive and rigorous clinical trials are lacking. Results may be affected by dosage, duration, and type of bacterial strains; host genotype; immune status; and endogenous microbiota. Nevertheless, probiotic supplements are currently widely used by pwMS (121). A clinical study evaluating the effect of probiotic mix on patients with MS and CIS is ongoing (NCT04038541).

Products derived from the gut microbiota. Preclinical and clinical investigations into the therapeutic potential of microbial products, particularly SCFAs, are currently ongoing. Increase in SCFA levels is commonly achieved through either high-fiber diet or direct SCFA supplementation (135). While SCFA supplementation has shown efficacy in reducing EAE clinical severity and inflammation, high-fiber diet produces more varying results $(20,136,137)$. Park and collaborators 
Table 4. Clinical trials testing gut-based therapies in pwMS

\begin{tabular}{|c|c|c|}
\hline Intervention & Ref. & Study details \\
\hline \multirow[t]{3}{*}{ Antibiotics } & 115 & $\begin{array}{l}\text { Open-label trial: } n=15 \text { pwMS with breakthrough disease activity were treated } \\
\text { with IFN- } \beta 1 \text { a and } 100 \mathrm{mg} \text { of doxycycline daily for } 4 \text { months }\end{array}$ \\
\hline & 116 & $\begin{array}{l}\text { Double-blind clinical trial: } n=60 \text { pwMS with breakthrough disease were treated with } \\
44 \mu \text { g subcutaneous IFN- } \beta 1 \text { la } 3 \text { times a week or } 30 \mu \text { intramuscular IFN- } \beta 1 \text { la once a } \\
\text { week plus } 100 \text { mg of doxycycline daily for } 6 \text { months }\end{array}$ \\
\hline & 117 & $\begin{array}{l}\text { Randomized placebo-controlled study: } n=142 \text { subjects diagnosed with CIS } \\
\text { assigned to receive either minocycline or placebo for } 24 \text { months }\end{array}$ \\
\hline \multirow[t]{3}{*}{ Probiotics } & 132 & $\begin{array}{l}\text { Randomized double-blind, placebo-controlled study: } n=60 \text { (30/group) } \\
\text { pwMS ( } 18-55 \text { years of age) with EDSS } \leq 4.5 \text { receiving a probiotic mixture } \\
\text { containing Lactobacillus acidophilus (L. acidophilus), Lactobacillus casei (L. casei), } \\
\text { Bifidobacterium bifidum (B. bifidum), and Lactobacillus fermentum (L. fermentum) } \\
\text { (each } 2 \times 10^{9} \mathrm{CFU} / \mathrm{g} \text { ) for } 12 \text { weeks }\end{array}$ \\
\hline & 133 & $\begin{array}{l}\text { Randomized, double-blind, placebo-controlled clinical trial: } n=40 \text { pwMS assigned } \\
\text { to receive either } L \text {. acidophilus, } L \text {. casei, } B \text {. bifidum, and } L \text {. fermentum }\left(2 \times 10^{9} \mathrm{CFU} / \mathrm{g}\right. \\
\text { each; } n=20) \text { or placebo }(n=20) \text { for } 12 \text { weeks }\end{array}$ \\
\hline & 168 & $\begin{array}{l}\text { Case-control study: } n=9 \text { RRMS patients and } 13 \mathrm{HCs} \text { treated with oral LBS } \\
\text { ( } 4 \text { strains of Lactobacillus, } 3 \text { strains of Bifidobacterium, and } 1 \text { strain of } \\
\text { Streptococcus) twice daily for } 2 \text { months }\end{array}$ \\
\hline
\end{tabular}

$\begin{array}{lll}\begin{array}{l}\text { Gut-derived } \\ \text { bacterial products }\end{array} & 21 & n=179 \text { pwMS and } 68 \text { HCs received oral propionic acid for } 14 \text { days } \\ \text { FMT } & 142 & \text { Case report on 1SPMS (EDSS 6) patient receiving an FMT. Ten-year follow-up } \\ & 141 & \text { Single-arm, nonrandomized, time series, single-subject study }\end{array}$

Dietary restriction

143

Randomized, double-blind, placebo-controlled clinical trial: $n=60$ participants assigned to ketogenic diet $(n=20)$, fasting mimicking diet for 7 days followed by Mediterranean diet $(n=20)$, or control $(n=20)$ for 6 months

151 Prospective cross-sectional study: $n=218$ RRMS patients who were able to fast during Ramadan

152 Randomized, double-blind, placebo-controlled clinical trial: $n=36$ RRMS randomly assigned to daily caloric restriction, intermittent caloric restriction, or control for 8 weeks

144 Randomized, double-blind, placebo-controlled clinical trial: $n=17$ RRMS enrolled during a relapse, randomly assigned to IF or control for 15 days

\section{Results}

Improved EDSS score and reduced gadolinium-enhancing lesions at MRI

$\downarrow$ relapse rate and improved EDSS score

$\downarrow$ risk of conversion to MS at 6 months

Improved plasma levels of CRP, nitric oxide metabolites, and MDA $\downarrow$ serum insulin and ameliorated insulin resistance parameters Improved EDSS, Beck depression inventory, general health questionnaire, and depression anxiety and stress scale

$\downarrow$ expression of IL-8 and TNF- $\alpha$ in PBMCs

Changes in the overall microbial community structure

$\downarrow$ abundance of several KEGG pathways

$\downarrow$ frequency of intermediate monocytes

$\downarrow$ expression of HLADQA1 and proinflammatory genes in peripheral monocytes

$\uparrow$ Treg induction and enhancement of Treg function Imbalance between Treg and Th17 cells in pwMS was restored

EDSS score stabilized

$\uparrow$ Firmicutes/Bacteroides and Prevotellaceae/Bacteroides ratio

$\uparrow$ abundance of Faecalibacterium prausnitzii (F. prausnitzii)

$\uparrow$ SCFA-genomic pathway

$\uparrow$ serum BDNF levels

Ameliorated gait metrics

Dietary restriction

Well tolerated

$\uparrow$ in MS-related quality of life

$\downarrow$ disability

$\uparrow$ cognitive domain score at the MFIS and physical and mental health composites at the QOL scores

Weight loss

$\uparrow$ emotional well-being

Weight loss

$\downarrow$ leptin and modulated gut microbiome composition

CIS, clinically isolated syndrome; CRP, C-reactive protein; KEGG, Kyoto Encyclopedia of Genes and Genomes; MDA, malondialdehyde; MFIS, Modified Fatigue Impact Scale; QOL, quality of life; SPMS, secondary progressive MS.

unveiled a possible dual role of SCFAs during CNS inflammation, whereby supplementation with a mix of propionate, butyrate, and acetate dampened EAE by increasing IL-10-producing T cells, but also induced inflammatory $\mathrm{T}$ cells through the GPCRs GPR 41 and -43 (20). Additionally, propionate supplementation before EAE induction, but not at disease onset, ameliorated disease and increased Tregs in the intestinal LP (136). In the cuprizone model of CNS demyelination, butyrate treatment for 1 week before commencing toxin administration decreased demyelination and enhanced remyelination (138). Administration of an SCFA mix via drinking water in GF mice in steady-state conditions rescued microglia defects associated with the absence of gut microbiota and gut-derived metabolites similarly to that observed in GF mice recolonized with live gut bacteria (139).
A recent clinical trial tested the effects of propionate supplementation for 2 weeks, as an add-on therapy in 91 pwMS and 24 HCs. Propionate lowered Th17 cells and increased Tregs and their in vitro suppressive capacity in an IL-10-dependent manner, and the effects were more pronounced in pwMS (21). Longitudinal data in 97 pwMS who underwent propionate supplementation for at least 1 year showed similar immunological changes, with a decrease in annualized relapse rate, disability stabilization, and a decrease in brain atrophy (21).

Besides SCFAs, other gut-derived metabolites have been studied as possible modulators of neuroinflammation. Bhargava and collaborators recently demonstrated that TUDCA administration reduced EAE clinical severity and could modulate astrocyte and 
microglia polarization in vitro (19). To the best of our knowledge, studies testing bile acids in humans have not been published yet, but an ongoing RCT is evaluating safety and tolerability of TUDCA in people with progressive MS (ClinicalTrials.gov NCT03423121).

To conclude, initial studies on SCFA supplementation are promising. While there are no known side effects, adherence to supplementation is impaired by butyrate's poor palatability. However, more randomized studies testing SCFAs or other gut bacterial products in pwMS are necessary to draw any conclusion.

Fecal microbiota transplantation. Given the condition of dysbiosis observed in pwMS, FMT has been proposed as a possible approach to reestablish a balanced gut microbiota. In EAE, FMT from naive into immunized mice resulted in reduced microglia and astrocyte activation, lower BBB leakage, and reduced demyelination and axonal loss (140). In a case study, a patient with MS who received daily FMTs from five donors displayed increased relative abundance of $F$. prusnitzii and levels of propionate, butyrate, and BDNF and decreased proinflammatory cytokines in the weeks following the transplant. Clinically, the patient demonstrated an improvement in balance and walking capacity (141). Another report described clinical stability during a 10-year follow-up in a patient with secondary progressive MS who underwent an FMT procedure to treat recurrent $C$. difficile infections (142).

In summary, there is insufficient evidence to suggest a clinical benefit of FMT in pwMS, and several clinical trials are ongoing (ClinicalTrials.gov NCT04096443, NCT03594487, NCT04150549). Difficulties in implementing this approach arise from lack of consensus on variables such as donor selection, fecal matter processing, route of administration, recipient premedication, and frequency of transplant. Such variables also create challenges when comparing outcomes from different studies.

Diet. Manipulating calorie intake and diet composition is another way to modulate CNS autoimmunity through changing gut microbial ecology and possibly other GBA components. Dietary interventions such as dietary restriction (DR), ketogenic diet, high-fiber and high-fat diets (143-146), and Paleolithic diet (147) have been tested in MS models and pwMS, but each diet's effects on gut microbiota have not been extensively investigated. $\mathrm{DR}$, defined as the chronic or intermittent reduction of calorie intake without malnutrition, has antiinflammatory effects and can ameliorate EAE through multiple mechanisms, including effects on the gut microbiota (148). Chronic or intermittent DR increases gut microbiota richness and alters its composition, as extensively reviewed recently (148). Lifelong DR in mice is known to change gut microbiota structure with enrichment of antiinflammatory bacteria strains, such as the genus Lactobacillus, accompanied by reduced serum proinflammatory endotoxin load from the gut (149). IF (24-hour fasting-feeding cycles) prior to disease induction leads to EAE attenuation, which is associated with changes in gut microbiota richness and composition $(144,150)$. Notably, disease protection is transferred by gut microbiota transplantation from IF mice into newly immunized recipients, suggesting the role of the gut microbiota in mediating DR's beneficial actions in EAE (144). DR could also act on other GBA components by activating the endocrine (e.g., increasing steroid hormone levels) and autonomic (e.g., activating the parasympathetic system) systems, which could contribute to its effects in reducing neuroinflamma- tion, as recently reviewed (148). Several clinical trials in pwMS also tested DR, reporting positive effects on measures of fatigue and emotional health $(143,151,152)$. In an RCT, 15 days of intermittent energy restriction versus ad libitum diet in 16 pwMS having a relapse was well tolerated and reduced levels of the proinflammatory adipokine leptin without altering levels of adiponectin. In this study, DR effects on the gut microbiota included enrichment of Faecalibacterium, Lachnospiraceae incertae sedis, and Blautia (all bacteria associated with immunomodulatory effects) while Faecalibacterium was strongly correlated with adiponectin levels (144). A longer trial in a larger cohort of pwMS is currently ongoing (ClinicalTrials.gov NCT03539094).

Modification of diet composition also modifies the gut microbiota and has been shown to interfere with CNS autoimmunity. As mentioned above, high-fiber diet can promote SCFA production by the gut microbiota. A clinical trial analyzing the effects of high fiber supplementation in a cohort of pwMS is ongoing (ClinicalTrials.gov NCT04574024). A recent study demonstrated that eliminating dietary tryptophan can produce a protective effect in EAE. Dietary tryptophan is necessary to prime MOG-reactive T cells to induce EAE, and a tryptophan-free diet is sufficient to completely prevent the disease (153). Interestingly, a group of vancomycin-resistant commensal bacteria, including $L$. reuteri, could catalyze the conversion of dietary tryptophan into AHR agonists capable of crossing the $\mathrm{BBB}$, suppressing astrocytic inflammatory profiles and improving EAE (113). In another recent study, dietary methionine restriction resulted in impaired $\mathrm{CD} 4^{+} \mathrm{T}$ cell activation and protection against EAE, since methionine is a critical component for Th17 cell proliferation and differentiation (154).

Targeting epithelial integrity. Studies focusing on possible therapeutic strategies to target epithelial integrity in MS or its animal model are lacking. However, in other inflammatory diseases, this approach seems promising. For instance, phosphatidylcholine constitutes a large proportion of phospholipids in the mucus layer of the intestine. Small studies on ulcerative colitis showed how phosphatidylcholine supplementation was more effective than placebo in reaching clinical remission through enhancing barrier integrity (155). While this remains untested in patients with MS, it provides a potential area to be explored.

\section{Effects of current MS treatments on the GBA}

Disease-modifying treatments (DMTs) and immunosuppressive drugs currently used to treat MS can have some effects on the GBA (Figure 1). Therapy-induced changes in the gut microbiota (e.g., favoring microbes with antiinflammatory properties) could possibly contribute to their clinical efficacy, even though these aspects have not been well investigated. A recent cross-sectional study analyzed the effects of glatiramer acetate (GA) and DMF in 168 pwMS; while they did not change the overall microbial community structure compared with untreated pwMS, both impacted the abundance of several genera, with the most significant effect of DMF being on the order of Clostridiales (156). In contrast, a pilot study using delayed-release DMF found no statistically significant effects on the gut microbiota (157). Other studies also reported changes in gut microbiota composition after GA or IFN- $\beta$ treatment, such as increased abundance of the genera Prevotella and Sutterella, with overall effects that tended to normalize some of the alterations observed in pwMS compared with 
HCs $(13,15)$. In EAE, the immunomodulatory effect of type I IFNs on CNS autoimmunity was linked to the induction of the transcription factor AHR, which was subsequently activated by microbially derived metabolites of tryptophan and resulted in antiinflammatory effects within the CNS. However, this study did not demonstrate a direct effect of treatment with type I IFNs on gut microbiota composition (113). Notably, both fingolimod and IFN- $\beta$ have gut barrier-stabilizing properties by promoting intestinal barrier integrity, partly by regulating TJ proteins $(55,158)$. Natalizumab, an anti- $\alpha 4$ integrin $\mathrm{mAb}$, modulates lymphocyte trafficking and inflammation across both the $\mathrm{BBB}$ and gut epithelia and has been shown to reduce the severity of inflammatory bowel disease (159).

Immunosuppressive drugs could affect the gut microbiota's function and composition. In nonhuman primates, alterations in gut microbiota composition and the immune system were described after treatment with alemtuzumab. Alemtuzumab induces profound systemic lymphocyte depletion that is also observed at the intestinal level (160). Lymphocyte depletion is associated with decreased abundance of Lactobacillus species and Weissella cibaria, decreased fungal diversity, and expansion of specific phylotypes, including Candida albicans $(161,162)$.

Steroids are commonly used to treat MS symptoms during a relapse, but little is known about the effect of steroid treatment on gut microbiota in pwMS. In mice, short- and long-term treatment with dexamethasone increased the abundance of Actinobacteria, Bifidobacterium, and Lactobacillus (163). As underlined above, pwMS show an overactivation of the HPA axis with higher levels of cortisol in the CSF compared with HCs (25). The activation of the HPA axis, associated with increased cortisol levels, can alter gut permeability, increasing circulating levels of immunostimulatory molecules (164).

Symptomatic treatments, such as drugs used for motor and/or psychiatric symptoms in pwMS, may also influence gut microbiota composition and could contribute to clinical efficacy. For example, antidepressant use can alter gut microbiota composition both directly and indirectly through modulation of neurotransmitter levels. The potential antimicrobial activity has been demonstrated for various classes of antidepressant drugs, such as tricyclics and selective serotonin reuptake inhibitors $(165,166)$, although the exact effect on gut flora composition is not clear. Similarly, a drug combining delta-9-tetrahydrocannabinol and cannabidiol, which is commonly used to treat muscle spasticity in pwMS, has been shown to reduce the levels of proinflammatory cytokines and the relative abundance of A. muciniphila in EAE (167).

\section{Conclusions}

Rapidly growing evidence supports the GBA's role in MS pathogenesis, with the gut microbiome as a critical player. However, more research is needed to better clarify molecular pathways connecting gut and brain functions and how they impact CNS autoimmunity. Interventions including FMT, probiotics, antibiotics, and diet that modulate the GBA have been actively investigated in preclinical models and to some extent also in clinical settings. Despite promising immunomodulatory effects of these therapies, only limited data exist on their impacts on neurodegenerative disease mechanisms. Nevertheless, we believe that in the future, therapies targeting the gut microbiota may be beneficial as an add-on treatment to already approved DMTs because they could potentially act synergistically. Some of these gut-based approaches (e.g., probiotic supplements) are already often implemented by pwMS because they are easily accessible as over-the-counter medications. While they do not have major side effects, the lack of appropriate regulatory organizations to control different preparations underlines the necessity for more controlled studies.

Looking ahead, microbiome-associated therapeutics will likely serve as an important component for precision medicine in MS. This will require a comprehensive understanding of patients' distinct gut microbial composition and function and thorough characterization of microbial interaction with the host immune system and other GBA components. This novel approach could also assist in monitoring response to treatments and may greatly benefit people suffering from this debilitating disease.

\section{Acknowledgments}

We thank members of our laboratories for helpful discussions. The authors apologize for the omission of relevant citations owing to space constraints. The work performed by our group and cited in this review was supported by grants R01 NS102633-01 to LP and YZ from the National Institute of Neurological Disorders and Stroke/NIH, by the Office of the Assistant Secretary of Defense for Health Affairs, through the Multiple Sclerosis Research Program under award W81XWH-14-1-0156; by the Leon and Harriet Felman Fund for Human MS Research; and by Fondazione Italiana Sclerosi Multipla (FISM; 2014/R/15) cofinanced with the "5 per mille" public funding. LG was supported by the Italian Multiple Sclerosis Society research fellowship (FISM 2018/B/1) and the National Multiple Sclerosis Society Post-Doctoral Fellowship (FG-1907-34474). CC was supported by the National MS Society Career Transition Fellowship (TA-1805-31003) and by the Whitelaw Terry, Jr./Valerie Terry funds.

Address correspondence to: Laura Piccio, Brain and Mind Centre, School of Medical Sciences, University of Sydney, 94 Mallett St., Camperdown, New South Wales 2050, Australia. Phone: 61.2.93510717; Email: laura.piccio@sydney.edu.au.
1. Braniste $\mathrm{V}$, et al. The gut microbiota influences blood-brain barrier permeability in mice. Sci Transl Med. 2014;6(263):263ra158.

2. Hoban AE, et al. Regulation of prefrontal cortex myelination by the microbiota. Transl Psychiatry. 2016;6(4):e774.

3. Sudo N, et al. Postnatal microbial colonization programs the hypothalamic-pituitary-adrenal system for stress response in mice. J Physiol. 2004;558(pt 1):263-275.
4. Bercik $\mathrm{P}$, et al. The intestinal microbiota affect central levels of brain-derived neurotropic factor and behavior in mice. Gastroenterology. 2011;141(2):599-609.

5. Heijtz RD, et al. Normal gut microbiota modulates brain development and behavior. Proc Natl Acad Sci U S A. 2011;108(7):3047-3052.

6. Carlson AL, et al. Infant gut microbiome associated with cognitive development. Biol Psychiatry. 2018;83(2):148-159.
7. Gao W, et al. Gut microbiome and brain functional connectivity in infants-a preliminary study focusing on the amygdala. Psychopharmacology (Berl). 2019;236(5):1641-1651.

8. Klingelhoefer L, Reichmann H. Pathogenesis of Parkinson disease - the gut-brain axis and environmental factors. Nat Rev Neurol. 2015;11(11):625-636.

9. Kadowaki A, Quintana FJ. The gut-CNS axis in multiple sclerosis. Trends Neurosci. 
2020;43(8):622-634.

10. Jiang $\mathrm{C}$, et al. The gut microbiota and Alzheimer's disease. JAlzheimers Dis. 2017;58(1):1-15.

11. Wallin MT, et al. The prevalence of multiple sclerosis in the United States: a population-based healthcare database approach. Neurology. 2019;92(10):e1029-e1040.

12. Ascherio A. Environmental factors in multiple sclerosis. Expert Rev Neurother. 2013;13(suppl 12):3-9.

13. Jangi S, et al. Alterations of the human gut microbiome in multiple sclerosis. Nat Commun. 2016;7:12015.

14. Chen J, et al. Multiple sclerosis patients have a distinct gut microbiota compared to healthy controls. Sci Rep. 2016;6:28484.

15. Cantarel BL, et al. Gut microbiota in multiple sclerosis: possible influence of immunomodulators. J Investig Med. 2015;63(5):729-734.

16. Berer K, et al. Gut microbiota from multiple sclerosis patients enables spontaneous autoimmune encephalomyelitis in mice. Proc Natl Acad Sci U S A. 2017;114(40):10719-10724

17. Cekanaviciute E, et al. Gut bacteria from multiple sclerosis patients modulate human T cells and exacerbate symptoms in mouse models. Proc Natl Acad Sci U S A. 2017;114(40):10713-10718.

18. Tremlett $\mathrm{H}$, et al. Gut microbiota in early pediatric multiple sclerosis: a case-control study. Eur J Neurol. 2016;23(8):1308-1321.

19. Bhargava $\mathrm{P}$, et al. Bile acid metabolism is altered in multiple sclerosis and supplementation ameliorates neuroinflammation. JClin Invest. 2020;130(7):3467-3482.

20. Park J, et al. Bidirectional regulatory potentials of short-chain fatty acids and their G-protein-coupled receptors in autoimmune neuroinflammation. Sci Rep. 2019;9(1):8837.

21. Duscha A, et al. Propionic acid shapes the multiple sclerosis disease course by an immunomodulatory mechanism. Cell. 2020;180(6):1067-1080.

22. Nouri M, et al. Intestinal barrier dysfunction develops at the onset of experimental autoimmune encephalomyelitis, and can be induced by adoptive transfer of auto-reactive T cells. PLOS One. 2014;9(9):e106335.

23. Mirza A, Mao-Draayer Y. The gut microbiome and microbial translocation in multiple sclerosis. Clin Immunol. 2017;183:213-224.

24. Buscarinu MC, et al. The contribution of gut barrier changes to multiple sclerosis pathophysiology. Front Immunol. 2019;10:1916.

25. Melief J, et al. HPA axis activity in multiple sclerosis correlates with disease severity, lesion type and gene expression in normal-appearing white matter. Acta Neuropathol. 2013;126(2):237-249.

26. Wunsch $\mathrm{M}$, et al. The enteric nervous system is a potential autoimmune target in multiple sclerosis. Acta Neuropathol. 2017;134(2):281-295.

27. Miyake S, et al. Dysbiosis in the gut microbiota of patients with multiple sclerosis, with a striking depletion of species belonging to clostridia XIVa and IV clusters. PLoS One. 2015;10(9):e1037429.

28. Cryan JF, et al. The gut microbiome in neurological disorders. Lancet Neurol. 2020;19(2):179-194.

29. Zhou X, et al. Household paired design reduces variance and increases power in multi-city gut microbiome study in multiple sclerosis. Mult Scler. 2021;27(3):366-379.
30. Chen $\mathrm{H}$, et al. Gut microbiota interventions with clostridium butyricum and norfloxacin modulate immune response in experimental autoimmune encephalomyelitis mice. Front Immunol. 2019;10:1662.

31. Cosorich I, et al. High frequency of intestinal TH17 cells correlates with microbiota alterations and disease activity in multiple sclerosis. Sci Adv. 2017;3(7):e1700492.

32. Atarashi $\mathrm{K}$, et al. Induction of colonic regulatory $\mathrm{T}$ cells by indigenous Clostridium species. Science. 2011;331(6015):337-341.

33. Johnson JL, et al. Polysaccharide A from the capsule of Bacteroides fragilis induces clonal CD4+ T cel expansion. J Biol Chem. 2015;290(8):5007-2014.

34. Ochoa-Repáraz J, et al. A polysaccharide from the human commensal Bacteroides fragilis protects against CNS demyelinating disease. Mucosal Immunol. 2010;3(5):487-495.

35. Ramakrishna C, et al. Bacteroides fragilis polysaccharide A induces IL-10 secreting $\mathrm{B}$ and $\mathrm{T}$ cells that prevent viral encephalitis. Nat Commun. 2019;10(1):2153.

36. Liu S, et al. Oral administration of miR-30d from feces of MS patients suppresses MS-like symptoms in mice by expanding Akkermansia muciniphila. Cell Host Microbe. 2019;26(6):779-794.

37. Takewaki D, et al. Alterations of the gut ecological and functional microenvironment in different stages of multiple sclerosis. Proc Natl Acad Sci US A. 2020;117(36):22402-22412.

38. Lewis K, et al. Enhanced translocation of bacteria across metabolically stressed epithelia is reduced by butyrate. Inflamm Bowel Dis. 2010;16(7):1138-1148.

39. Rothhammer V, et al. Microglial control of astrocytes in response to microbial metabolites. Nature. 2018;557(7707):724-728.

40. Corrêa-Oliveira R, et al. Regulation of immune cell function by short-chain fatty acids. Clin Transl Immunology. 2016;5(4):e73.

41. Pérez-Pérez S, et al. Acetate correlates with disability and immune response in multiple sclerosis. PeerJ. 2020;8:e10220.

42. Grant SM, Demorrow S. Bile acid signaling in neurodegenerative and neurological disorders. Int J Mol Sci. 2020;21(17):5982.

43. Lee $\mathrm{G}$, et al. Identification of altered metabolic pathways during disease progression in EAE mice via metabolomics and lipidomics. Neuroscience. 2019;416:74-87.

44. Chelakkot C, et al. Mechanisms regulating intestinal barrier integrity and its pathological implications. Exp Mol Med. 2018;50(8):1-9.

45. Okumura R, Takeda K. Roles of intestinal epithelial cells in the maintenance of gut homeostasis. Exp Mol Med. 2017;49(5):e338.

46. Lee B, et al. Tight junction in the intestinal epithelium: its association with diseases and regulation by phytochemicals. J Immunol Res. 2018;2018:2645465.

47. Fasano A. Zonulin and its regulation of intestinal barrier function: the biological door to inflammation, autoimmunity, and cancer. Physiol Rev. 2011;91(1):151-175.

48. González-Mariscal L, et al. Tight junction proteins. Prog Biophys Mol Biol. 2003;81(1):1-44.

49. Slifer ZM, Blikslager AT. The integral role of tight junction proteins in the repair of injured intesti- nal epithelium. Int J Mol Sci. 2020;21(3):972.

50. Tervonen A, et al. Structural dynamics of tight junctions modulate the properties of the epithelial barrier. PLoS One. 2019;14(4):e0214876.

51. Ulluwishewa D, et al. Regulation of tight junction permeability by intestinal bacteria and dietary components. J Nutr. 2011;141(5):769-776.

52. Landy J, et al. Tight junctions in inflammatory bowel diseases and inflammatory bowel disease associated colorectal cancer. World J Gastroenterol. 2016;22(11):3117-3126.

53. Antonini M, et al. How the interplay between the commensal microbiota, gut barrier integrity, and mucosal immunity regulates brain autoimmunity. Front Immunol. 2019;10:1937.

54. Buscarinu MC, et al. Altered intestinal permeability in patients with relapsing-remitting multiple sclerosis: a pilot study. Mult Scler. 2017;23(3):442-446.

55. Camara-Lemarroy CR, et al. Biomarkers of intestinal barrier function in multiple sclerosis are associated with disease activity. Mult Scler J. 2020;26(11):1340-1350.

56. Escribano BM, et al. Lipopolysaccharide binding protein and oxidative stress in a multiple sclerosis model. Neurotherapeutics. 2017;14(1):199-211.

57. Laman JD, et al. Bacterial peptidoglycan as a driver of chronic brain inflammation. Trends Mol Med. 2020;26(7):670-682.

58 . Kriesel JD, et al. Spectrum of microbial sequences and a bacterial cell wall antigen in primary demyelination brain specimens obtained from living patients. Sci Rep. 2019;9(1):1387.

59. Secher T, et al. Oral administration of the probiotic strain Escherichia coli Nissle 1917 reduces susceptibility to neuroinflammation and repairs experimental autoimmune encephalomyelitis-induced intestinal barrier dysfunction. Front Immunol. 2017;8:1096.

60. Paone P, Cani PD. Mucus barrier, mucins and gut microbiota: the expected slimy partners? Gut. 2020;69(12):2232-2243.

61. Derrien M, et al. Modulation of mucosal immune response, tolerance, and proliferation in mice colonized by the mucin-degrader Akkermansia muciniphila. Front Microbiol. 2011;2:166.

62. Gandy KAO, et al. The role of gut microbiota in shaping the relapse-remitting and chronic-progressive forms of multiple sclerosis in mouse models. Sci Rep. 2019;9(1):6923.

63. Freedman SN, et al. The "gut feeling": breaking down the role of gut microbiome in multiple sclerosis. Neurotherapeutics. 2018;15(1):109-125

64. Cox LM, et al. The gut microbiome in progressive multiple sclerosis. Ann Neurol. 2021;89(6):1195-1211.

65. Gurung M, et al. Role of gut microbiota in type 2 diabetes pathophysiology. EBioMedicine. 2020;51:102590.

66. Mowat AM, Agace WW. Regional specialization within the intestinal immune system. Nat Rev Immunol. 2014;14(10):667-685.

67. Round JL, Mazmanian SK. The gut microbiota shapes intestinal immune responses during health and disease. Nat Rev Immunol. 2009;9(5):313-323.

68. Smith K, et al. Use of axenic animals in studying the adaptation of mammals to their com- 
mensal intestinal microbiota. Semin Immunol. 2007;19(2):59-69.

69. Chung H, et al. Gut immune maturation depends on colonization with a host-specific microbiota. Cell. 2012;149(7):1578-1593.

70. Ivanov II, et al. Induction of intestinal Th17 cells by segmented filamentous bacteria. Cell. 2009;139(3):485-498.

71. Eisenstein EM, Williams CB. The Treg/Th17 cell balance: a new paradigm for autoimmunity. Pediatr Res. 2009;65(5 pt 2):26R-31R.

72. Sakaguchi S, et al. Regulatory T cells and immune tolerance. Cell. 2008;133(5):775-787.

73. Cheng H, et al. The Th17/Treg cell balance: a gut microbiota-modulated story. Microorganisms. 2019;7(12):583.

74. Omenetti S, Pizarro TT. The Treg/Th17 axis: a dynamic balance regulated by the gut microbiome. Front Immunol. 2015;6:639.

75. Regen T, et al. IL-17 controls central nervous system autoimmunity through the intestinal microbiome. Sci Immunol. 2021;6(56):eaaz6563.

76. Lee YK, et al. Proinflammatory T-cell responses to gut microbiota promote experimental autoimmune encephalomyelitis. Proc Natl Acad Sci US A. 2011;108(suppl 1):4615-4622.

77. Yokote $\mathrm{H}$, et al. NKT cell-dependent amelioration of a mouse model of multiple sclerosis by altering gut flora. Am J Pathol. 2008;173(6):1714-1723.

78. Duc D, et al. Disrupting Myelin-specific Th17 cell gut homing confers protection in an adoptive transfer experimental autoimmune encephalomyelitis. Cell Rep. 2019;29(2):378-390.

79. Berer K, et al. Selective accumulation of pro-inflammatory $\mathrm{T}$ cells in the intestine contributes to the resistance to autoimmune demyelinating disease. PLoS One. 2014;9(2):e87876.

80. James KR, et al. Distinct microbial and immune niches of the human colon. Nat Immunol. 2020;21(3):343-353.

81. Bos NA, et al. Serum immunoglobulin levels and naturally occurring antibodies against carbohydrate antigens in germ-free BALB/c mice fed chemically defined ultrafiltered diet. Eur J Immunol. 1989;19(12):2335-2339.

82. Rojas OL, et al. Recirculating intestinal IgA-producing cells regulate neuroinflammation via IL-10. Cell. 2019;176(3):610-624.

83. Pröbstel AK, et al. Gut microbiota-specific iga+ B cells traffic to the CNS in active multiple sclerosis. Sci Immunol. 2020;5(53):eabc7191.

84. Langley JN. The autonomic nervous system. Brain. 1903;26(1):1-26.

85. Rao M, Gershon MD. The bowel and beyond: the enteric nervous system in neurological disorders. Nat Rev Gastroenterol Hepatol. 2016;13(9):517-528.

86. Collins J, et al. Intestinal microbiota influence the early postnatal development of the enteric nervous system. Neurogastroenterol Motil. 2014;26(1):98-107.

87. Hosoi T, et al. Novel pathway for LPS-induced afferent vagus nerve activation: possible role of nodose ganglion. Auton Neurosci. 2005;120(1-2):104-107.

88. Strader AD, Woods SC. Gastrointestinal hormones and food intake. Gastroenterology. 2005;128(1):175-191.

89. Tsuchida Y, et al. Neuronal stimulation with 5-hydroxytryptamine 4 receptor induces anti-in- flammatory actions via $\alpha 7 \mathrm{nACh}$ receptors on muscularis macrophages associated with postoperative ileus. Gut. 2011;60(5):638-647.

90. Di Bari M, et al. Dysregulated homeostasis of acetylcholine levels in immune cells of RR-multiple sclerosis patients. Int JMol Sci. 2016;17(12):2009.

91. Reale M, et al. Relation between pro-inflammatory cytokines and acetylcholine levels in relapsing-remitting multiple sclerosis patients. Int J Mol Sci. 2012;13(10):12656-12664.

92. Nizri E, et al. Anti-inflammatory properties of cholinergic up-regulation: a new role for acetylcholinesterase inhibitors. Neuropharmacology. 2006;50(5):540-547.

93. Zhou L, et al. Acetylcholine regulates the development of experimental autoimmune encephalomyelitis via the CD4+ cells proliferation and differentiation. Int J Neurosci. 2020;130(8):788-803.

94. Carrasco GA, Van de Kar LD. Neuroendocrine pharmacology of stress. Eur J Pharmacol. 2003;463(1-2):235-272.

95. Czimmer J, et al. Urocortin 2 acts centrally to delay gastric emptying through sympathetic pathways while $\mathrm{CRF}$ and urocortin 1 inhibitory actions are vagal dependent in rats. Am J Physiol Gastrointest Liver Physiol. 2006;290(3):G511-G518.

96. Zheng G, et al. Corticosterone mediates stress-related increased intestinal permeability in a region-specific manner. Neurogastroenterol Motil. 2013;25(2):e127-e139.

97. Zen M, et al. The kaleidoscope of glucorticoid effects on immune system. Autoimmun Rev. 2011;10(6):305-310.

98. Farzi A, et al. Gut microbiota and the neuroendocrine system. Neurotherapeutics. 2018;15(1):5-22.

99. Crumeyrolle-Arias M, et al. Absence of the gut microbiota enhances anxiety-like behavior and neuroendocrine response to acute stress in rats. Psychoneuroendocrinology. 2014;42:207-217.

100. Bravo JA, et al. Ingestion of Lactobacillus strain regulates emotional behavior and central GABA receptor expression in a mouse via the vagus nerve. Proc Natl Acad Sci U S A . 2011;108(38):16050-16055.

101. Neufeld KM, et al. Reduced anxiety-like behavior and central neurochemical change in germ-free mice. Neurogastroenterol Motil. 2011;23(3):255-264.

102. Fassbender K, et al. Mood disorders and dysfunction of the hypothalamic-pituitary-adrenal axis in multiple sclerosis: association with cerebral inflammation. Arch Neurol. 1998;55(1):66-72.

103. Gold SM, et al. Hypothalamo-pituitary-adrenal axis activity predicts disease progression in multiple sclerosis. J Neuroimmunol. 2005;165(1-2):186-191.

104. Huitinga I, et al. Impaired hypothalamus-pituitary-adrenal axis activity and more severe multiple sclerosis with hypothalamic lesions. Ann Neurol. 2004;55(1):37-45.

105. Grasser A, et al. Heterogeneity of hypothalamicpituitary-adrenal system response to a combined dexamethasone-CRH test in multiple sclerosis. Exp Clin Endocrinol Diabetes. 1996;104(1):31-37.

106. Heesen C, et al. Correlates of cognitive dysfunction in multiple sclerosis. Brain Behav Immun. 2010;24(7):1148-1155.

107. Ogbonnaya ES, et al. Adult hippocampal neurogenesis is regulated by the microbiome. Biol Psychiatry. 2015;78(4):e7-e9.

108. Ochoa-Repáraz J, et al. Role of gut commensal microflora in the development of experimental autoimmune encephalomyelitis. JImmunol. 2009;183(10):6041-6050.

109. Miyauchi E, et al. Gut microorganisms act together to exacerbate inflammation in spinal cords. Nature. 2020;585(7823):102-106.

110. Colpitts SL, et al. A bidirectional association between the gut microbiota and CNS disease in a biphasic murine model of multiple sclerosis. Gut Microbes. 2017;8(6):561-573.

111. Gonzalez CG, et al. Latent-period stool proteomic assay of multiple sclerosis model indicates protective capacity of host-expressed protease inhibitors. Sci Rep. 2019;9(1):12460.

112. Mestre L, et al. Manipulation of gut microbiota influences immune responses, axon preservation, and motor disability in a model of progressive multiple sclerosis. Front Immunol. 2019;10:1374.

113. Rothhammer V, et al. Type I interferons and microbial metabolites of tryptophan modulate astrocyte activity and central nervous system inflammation via the aryl hydrocarbon receptor. Nat Med. 2016;22(6):586-597.

114. McMurran CE, et al. The microbiota regulates murine inflammatory responses to toxin-induced CNS demyelination but has minimal impact on remyelination. Proc Natl Acad Sci U S A. 2019;116(50):25311-25321.

115. Minagar A, et al. Combination therapy with interferon beta-1a and doxycycline in multiple sclerosis: an open-label trial. Arch Neurol. 2008;65(2):199-204.

116. Mazdeh M, Mobaien AR. Efficacy of doxycycline as add-on to interferon beta-1a in treatment of multiple sclerosis. Iran J Neurol. 2012;11(2):70-73.

117. Metz LM, et al. Trial of minocycline in a clinically isolated syndrome of multiple sclerosis. $\mathrm{N} \mathrm{EnglJ}$ Med. 2017;376(22):2122-2133.

118. Kirby T, Ochoa-Repáraz J. The gut microbiome in multiple sclerosis: a potential therapeutic avenue. Med Sci (Basel). 2018;6(3):69.

119. Ross CL, et al. Structural and functional changes within the gut microbiota and susceptibility to Clostridium difficile infection. Anaerobe. 2016;41:37-43.

120. Suez J, et al. The pros, cons, and many unknowns of probiotics. Nat Med. 2019;25(5):716-729.

121. Morshedi M, et al. Immunomodulatory and anti-inflammatory effects of probiotics in multiple sclerosis: a systematic review. J Neuroinflammation. 2019;16(1):231.

122. He B, et al. Lactobacillus reuteri reduces the severity of experimental autoimmune encephalomyelitis in mice by modulating gut microbiota. Front Immunol. 2019;10:385.

123. Yamashita M, et al. Lactobacillus helveticus SBT2171 attenuates experimental autoimmune encephalomyelitis in mice. Front Microbiol. 2018;8:2596.

124. Salehipour Z, et al. Bifidobacterium animalis in combination with human origin of Lactobacillus plantarum ameliorate neuroinflammation in experimental model of multiple sclerosis by altering CD4+ T cell subset balance. Biomed Pharmacother. 2017;95:1535-1548.

125. Lavasani S, et al. A novel probiotic mixture exerts a therapeutic effect on experimental 
autoimmune encephalomyelitis mediated by IL-10 producing regulatory T cells. $P$ LoS One. 2010;5(2):e9009.

126. Ezendam J, van Loveren H. Lactobacillus casei Shirota administered during lactation increases the duration of autoimmunity in rats and enhances lung inflammation in mice. Br J Nutr. 2008;99(1):83-90.

127. Maassen CBM, et al. Reduced experimental autoimmune encephalomyelitis after intranasal and oral administration of recombinant lactobacilli expressing myelin antigens. Vaccine. 2003;21(32):4685-4693.

128. Consonni A, et al. Administration of bifidobacterium and lactobacillus strains modulates experimental myasthenia gravis and experimental encephalomyelitis in Lewis rats. Oncotarget. 2018;9(32):22269-22287.

129. Montgomery TL, et al. Interactions between host genetics and gut microbiota determine susceptibility to CNS autoimmunity. Proc Natl Acad Sci U S A. 2020;117(44):27516-27527.

130. Mangalam A, et al. Human gut-derived commensal bacteria suppress CNS inflammatory and demyelinating disease. Cell Rep. 2017;20(6):1269-1277.

131. Mestre L, et al. How oral probiotics affect the severity of an experimental model of progressive multiple sclerosis? Bringing commensal bacteria into the neurodegenerative process. Gut Microbes. 2020;12(1):1813532.

132. Kouchaki E, et al. Clinical and metabolic response to probiotic supplementation in patients with multiple sclerosis: a randomized, double-blind, placebo-controlled trial. Clin Nutr. 2017;36(5):1245-1249.

133. Tamtaji OR, et al. The effects of probiotic supplementation on gene expression related to inflammation, insulin, and lipids in patients with multiple sclerosis: a randomized, double-blind, placebo-controlled trial.J Am Coll Nutr. 2017;36(8):660-665.

134. Tankou SK, et al. A probiotic modulates the microbiome and immunity in multiple sclerosis. Ann Neurol. 2018;83(6):1147-1161.

135. Wong JMW, et al. Colonic health: fermentation and short chain fatty acids. JClin Gastroenterol. 2006;40(3):235-243.

136. Haghikia A, et al. Dietary fatty acids directly impact central nervous system autoimmunity via the small intestine. Immunity. 2015;43(4):817-829.

137. Mizuno M, et al. The dual role of short fatty acid chains in the pathogenesis of autoimmune disease models. PLoS One. 2017;12(2):e0173032.

138. Chen T, et al. Butyrate suppresses demyelination and enhances remyelination. JNeuroinflamma- tion. 2019;16(1):165.

139. Erny D, et al. Host microbiota constantly control maturation and function of microglia in the CNS Nat Neurosci. 2015;18(7):965-977.

140.Li K, et al. Protection of fecal microbiota transplantation in a mouse model of multiple sclerosis. Mediators Inflamm. 2020;2020:2058272.

141. Engen PA, et al. Single-arm, non-randomized, time series, single-subject study of fecal microbiota transplantation in multiple sclerosis. Front Neurol. 2020;11:978.

142. Makkawi S, et al. Fecal microbiota transplantation associated with 10 years of stability in a patient with SPMS. Neurol Neuroimmunol NeuroInflammation. 2018;5(4):e459.

143. Choi IY, et al. A diet mimicking fasting promotes regeneration and reduces autoimmunity and multiple sclerosis symptoms. Cell Rep. 2016;15(10):2136-2146.

144. Cignarella F, et al. Intermittent fasting confers protection in CNS autoimmunity by altering the gut microbiota. Cell Metab. 2018;27(6):1222-1235.

145. Bahr LS, et al. Ketogenic diet and fasting diet as nutritional approaches in multiple sclerosis (NAMS): protocol of a randomized controlled study. Trials. 2020;21(1):3.

146.Timmermans S, et al. High fat diet exacerbates neuroinflammation in an animal model of multiple sclerosis by activation of the renin angiotensin system. J Neuroimmune Pharmacol. 2014;9(2):209-217.

147. Maxwell KF, et al. Lipid profile is associated with decreased fatigue in individuals with progressive multiple sclerosis following a diet-based intervention: results from a pilot study. PLoS One. 2019;14(6):e0218075.

148. Fontana L, et al. Effects of dietary restriction on neuroinflammation in neurodegenerative diseases. J Exp Med. 2021;218(2):e20190086.

149.Zhang C, et al. Structural modulation of gut microbiota in life-long calorie-restricted mice. Nat Commun. 2013;4:2163.

150. Kafami L, et al. Intermittent feeding attenuates clinical course of experimental autoimmune encephalomyelitis in C57BL/6 mice. Avicenna J Med Biotechnol. 2010;2(1):47-52.

151. Etemadifar M, et al. Effects of prolonged fasting on fatigue and quality of life in patients with multiple sclerosis. Neurol Sci. 2016;37(6):929-933.

152. Fitzgerald KC, et al. A survey of dietary characteristics in a large population of people with multiple sclerosis. Mult Scler Relat Disord. 2018;22:12-18.

153. Sonner JK, et al. Dietary tryptophan links encephalogenicity of autoreactive $\mathrm{T}$ cells with gut microbial ecology. Nat Commun. 2019;10(1):4877.
154. Roy DG, et al. Methionine metabolism shapes T helper cell responses through regulation of epigenetic reprogramming. Cell Metab. 2020;31(2):250-266

155. Stremmel W, et al. Mucosal protection by phosphatidylcholine. Dig Dis. 2012;30(suppl 3):85-91.

156.Sand IK, et al. Disease-modifying therapies alter gut microbial composition in MS. Neurol Neuroimmunol NeuroInflammation. 2018;6(1):e517.

157. Storm-Larsen C, et al. Gut microbiota composition during a 12 -week intervention with delayed-release dimethyl fumarate in multiple sclerosis - a pilot trial. Mult Scler J Exp Transl Clin. 2019;5(4):2055217319888767.

158. Long TM, et al. Enteropathogenic Escherichia coli inhibits type I interferon- and RNase L-mediated host defense to disrupt intestinal epithelial cell barrier function. Infect Immun. 2014;82(7):2802-2814

159. Bamias G, et al. Leukocyte traffic blockade as a therapeutic strategy in inflammatory bowel disease. Curr Drug Targets. 2013;14(12):1490-1500.

160. Li Q, et al. Influence of alemtuzumab on the intestinal Paneth cells and microflora in macaques. Clin Immunol. 2010;136(3):375-386.

161. Li Q, et al. Lymphocyte depletion after alemtuzumab induction disrupts intestinal fungal microbiota in cynomolgus monkeys. Transplantation. 2014;98(9):951-959.

162.Li QR, et al. Reciprocal interaction between intestinal microbiota and mucosal lymphocyte in cynomolgus monkeys after alemtuzumab treatment. Am J Transplant. 2013;13(4):899-910.

163. Huang EY, et al. Using corticosteroids to reshape the gut microbiome: implications for inflammatory bowel diseases. Inflamm Bowel Dis. 2015;21(5):963-972.

164. Cryan JF, Dinan TG. Mind-altering microorganisms: the impact of the gut microbiota on brain and behaviour. Nat Rev Neurosci. 2012;13(10):701-712.

165. Ayaz M, et al. Sertraline enhances the activity of antimicrobial agents against pathogens of clinical relevance. J Biol Res (Thessalon). 2015;21(1):4

166. Csiszar K, Molnar J. Mechanism of action of tricyclic drugs on Esherichia coli and Yersinia enterocolitica plasmid maintenance and replication. Anticancer Res. 1992;12(6B):2267-2272.

167. Al-Ghezi ZZ, et al. Combination of cannabinoids, delta-9-tetrahydrocannabinol (THC) and cannabidiol (CBD), mitigates experimental autoimmune encephalomyelitis (EAE) by altering the gut microbiome. Brain Behav Immun. 2019;82:25-35.

168. Tankou SK, et al. Investigation of probiotics in multiple sclerosis. Mult Scler. 2018;24(1):58-63. 